Check for updates

Cite this: RSC Adv., 2017, 7, 20192

Received 24th February 2017

Accepted 31st March 2017

DOI: $10.1039 / c 7 r a 02302 b$

rsc.li/rsc-advances

\section{Notably enhanced thermoelectric properties of lamellar polypyrrole by doping with $\beta$-naphthalene sulfonic acid}

\author{
Xianxian Tang, Taoxiang Liu, ${ }^{*}$ Han Li, Dongwang Yang, Liangjun Chen \\ and Xinfeng Tang (iD *
}

Polypyrrole (PPy) is a type of potential organic thermoelectric material that has attracted extensive attention in recent years. Its application is mainly limited by the relatively low intrinsic electrical conductivity and Seebeck coefficient, so it is necessary to find an effective way to improve the electrical transport properties of PPy. In this work, lamellar PPy doped with $\beta$-naphthalene sulfonic acid ( $\beta$-NSA) was synthesized chemically using ferric chloride $\left(\mathrm{FeCl}_{3}\right)$ as the oxidant, and the thermoelectric performance of $\beta$-NSA doped PPy was significantly enhanced. FTIR, XRD, Raman and FESEM analyses indicated that the presence of $\beta-\mathrm{NSA}$ and $\mathrm{FeCl}_{3}$ could effectively control the morphology of PPy to form a lamellar structure during the polymerization process, which improves the thermoelectric properties of PPy greatly. The highest thermoelectric figure of merit $Z T, 0.62 \times 10^{-3}$, was obtained with the molar ratio of monomer pyrrole (py) to $\beta$-NSA of $1.0: 0.45$ at $300 \mathrm{~K}$, and was 10 times higher than that of pure PPy. Furthermore, the TGA, elastic modulus, Vickers hardness results showed that the thermal stability and mechanical properties of $\beta$-NSA doped PPy was better than that of pure PPy.

\section{Introduction}

With the exhaustion of traditional energy sources such as coal and oil, and increasingly serious environmental pollution, there is urgent need to develop new energy conversion materials and clean energy to solve these problems. Thermoelectric (TE) conversion technology is capable of converting thermal energy into electrical energy directly using TE materials. ${ }^{1-3}$ In recent years, TE materials have attracted much attention in the world because of their lack of pollution, noise, and moving parts, their high reliability and other significant advantages. ${ }^{4-6}$ The performance of thermoelectric materials can be characterized by the dimensionless figure of merit $Z T=S^{2} \sigma T / \kappa$, where $S, \sigma, \kappa$ and $T$ are the Seebeck coefficient, electrical conductivity, thermal conductivity, and absolute temperature, respectively. ${ }^{7}$

Over the years, researches were mainly concentrated on inorganic thermoelectric materials due to the relatively high performance and good high-temperature stability. However, the high cost of raw materials and the complicated manufacturing as well as the poor processing performance limits its widespread use. ${ }^{8}$ In recent years, organic thermoelectric materials have been developed rapidly thanks to their abundant resources, low weight, low cost, low thermal conductivity, simple preparation process and flexible preparation of wearable

State Key Laboratory of Advanced Technology for Materials Synthesis and Processing, Wuhan University of Technology, Wuhan 430070, P. R. China. E-mail: tangxf@whut. edu.cn; cxzwut@126.com products with different shapes, which made it become a highprofile international research field. ${ }^{9-11}$ For example, poly $(3,4-$ ethylenedioxythiophene)/poly (styrenesulphonate) (PEDOT/ PSS), ${ }^{12-14}$ polyaniline (PANI) ${ }^{15,16}$ polypyrrole (PPy), ${ }^{17,18}$ and other organic thermoelectric materials have been studied extensively. ${ }^{19} \mathrm{Kim}$ et al. reported that the $Z T$ value of PEDOT reaches 0.42 at room temperature after doping with $\mathrm{PSS}^{20} \mathrm{Li}$ et al. fabricated a series of hydrochloric acid-doped PANI, found that the $Z T_{\max }$ achieves $2.67 \times 10^{-4}$ at $423 \mathrm{~K}$ when HCl-doping concentration is $1.0 \mathrm{M}^{21}$

In contrast with other conductive polymers, PPy has been widely reported in battery, super capacitor, electromagnetic shielding, biological sensors and so on ${ }^{22-25}$ in virtue of its non toxic, environmental protection, low thermal conductivity, good biocompatibility, good stability in air, ${ }^{26,27}$ and meanwhile these advantages also make PPy as a potential organic thermoelectric material. ${ }^{28,29}$ But the intrinsic electrical conductivity and Seebeck coefficient of PPy is relatively low, and its thermal stability is not good enough. To solve these issues, most researchers mainly focused on doping or combining with other materials. For example, Wang et al. successfully synthesized the composite of PPy and graphene nanosheets (GNs) with enhanced thermoelectric properties. The $Z T$ value increased with increasing GNs content and the maximum $Z T$ value of $2.80 \times 10^{-3}$ is about 210 times higher than that obtained from pure PPy. ${ }^{30}$ Wang et al. prepared PPy/multi-walled carbon nanotubes (MWCNTs) composite powders in the presence of ferric chloride $\left(\mathrm{FeCl}_{3}\right)$ as the oxidant, resulting in the surfaces of MWCNT coated with 
PPy nanoparticles. And the power factor of the PPy/MWCNTs composite is $2.079 \mu \mathrm{W} \mathrm{m}^{-1} \mathrm{~K}^{-2}$ when the MWCNTs content is $20 \%$, which is about 26 times as high as that of pure PPy. ${ }^{31}$ Shen et al. mainly studied the solubility of the $\beta$-NSA doped PPy in $m$ cresol, and the PPy-NSA prepared by in situ doped polymerization method with ammonium persulfate (APS) as the oxidant exhibited fibrillar morphology, which was different from the typical granular morphology of PPy doped with DBSA, HCl, MBSA or CSA, while the PPy/ $\beta$-NSA prepared by two-step method was also granular morphology. And the highest electrical conductivity of $\beta$-NSA doped PPy is $27 \mathrm{~S} \mathrm{~cm}^{-1}$ at room temperature. ${ }^{32}$ In another work, they investigated the solubility of the PPy doped with different sulfonic acid (BNSA, $\beta$-NSA, DBSA, CSA, MBSA and so on), and proposed that the solubility of PPy is connected with the solvating ability of sulfonic acid. They also studied the influences of the various polymerization medium (water, $\mathrm{CHCl}_{3}, \mathrm{CH}_{3} \mathrm{NO}_{2}$, THF) on the solubility and conductivity of PPy. ${ }^{33}$ Akinyeye et al. synthesized $\beta$-NSA doped PPy micro/ nanotubes, they pointed out that the stability of the conducting cationic polypyrrole intermediate in the polymerization process is favoured in acidic medium. And the acidic media provided by the $\beta$-NSA dopant will significantly influence the conductivity of PPy. ${ }^{34}$ What's more, $\beta$-NSA can ionize hydrophilic group of naphthalene sulfonic acid ion ( $\mathrm{NSA}^{-}$) in solution simultaneously, which may act as template to affect the micromorphology of PPy in the polymerization process, and affect the carrier transportation within intrachain and interchain. Thereby the electrical conductivity can be further improved. ${ }^{35}$

From the above, the microstructure of PPy has a great impact on its thermoelectric performance, and doping PPy with $\beta$-NSA may improve the thermoelectric performance of PPy. In this work, lamellar PPy doped with $\beta$-NSA was successfully prepared with various doping amount of $\beta$-NSA through the chemical oxidative polymerization method, using ferric chloride $\left(\mathrm{FeCl}_{3}\right)$ as the oxidant and the $\beta$-NSA as the dopant, respectively. The formation mechanism of lamellar structure and the thermoelectric properties of the prepared samples were studied.

\section{Experimental}

Pyrrole (99\%), $\beta$-naphthalene sulfonic acid ( $\beta$-NSA) (98\%), anhydrous ferric chloride $\left(\mathrm{FeCl}_{3}\right)(\mathrm{AR})$, anhydrous ethanol (AR) and deionized water were the main raw materials. All the chemical reagents were of analytical grade and without any further purification. Typically, $0.97 \mathrm{~g}$ pyrrole monomer was dissolved in $80 \mathrm{~mL}$ deionized water, then a certain amount of $\beta$ NSA was added into the above solution, the molar ratios of pyrrole monomer to $\beta$-NSA were designed as $1.0: 0.4,1.0: 0.45$, $1.0: 0.5,1.0: 0.55,1.0: 0.6$, respectively. After magnetic stirring for $30 \mathrm{~min}$ in ice bath $\left(<5^{\circ} \mathrm{C}\right)$, the oxidant solution obtained by dissolving $4.69 \mathrm{~g} \mathrm{FeCl}_{3}$ in $80 \mathrm{~mL}$ deionized water was slowly dropped into the above reaction system via pear-shaped funnel. The reaction was carried out under continuously stirring for $12 \mathrm{~h}$ in ice bath $\left(<5^{\circ} \mathrm{C}\right)$. After the polymerization, the resulting precipitates were centrifuged and washed with anhydrous ethanol for several times until colorless. Finally, as-prepared products were dried under vacuum at $50{ }^{\circ} \mathrm{C}$ for $24 \mathrm{~h}$. Pure PPy without $\beta$-NSA was also prepared under the similar procedure.

Fourier transform infrared spectroscopy (FTIR, NEXUS/ NEXUS, USA) measurements were conducted to test the characteristic absorption peaks of functional group, with the scanning range of $4000-400 \mathrm{~cm}^{-1}$. The X-ray diffraction (XRD) spectra of the all samples were checked on a PANalytical X'Pert Empyean Xray diffractometer with $\mathrm{Cu} \mathrm{K} \alpha(1.5406 \AA)$ with the generator voltage and the tube current to be $40 \mathrm{kV}$ and $40 \mathrm{~mA}$, and scanning was carried out in the $2 \theta$ range from 3 to 50 degrees with a scan step size of 0.02 degree. Raman spectra were recorded on a Raman spectrometer (NVIA/INVIA, China) with an excitation wavelength of $633 \mathrm{~nm}$. Field emission scanning electron microscopy (SEM, HITACHI SU8020, Japan) was employed to observe the microstructure of the powder sample. Comprehensive thermal analyzer (TG-DSC, STA449F3/STA449F3, Germany) was applied to investigate the thermal stability of the sample under nitrogen atmosphere at a heating rate of $10{ }^{\circ} \mathrm{C} \mathrm{min}^{-1}$. The electrical conductivity and the Seebeck coefficient of the samples were acquired on a commercial ZEM-3 system (Ulvac Sinku-Riko, Japan) in helium in the temperature range of $300-400 \mathrm{~K}$. The thermal conductivity of the sample was determined from $\kappa=$ $\lambda C_{\mathrm{p}} d$, where $\lambda$ is the thermal diffusivity acquired by the laser flash method (Netzsch LFA 457, Germany), $d$ is the density measured by the Archimedes method in alcohol, and $C_{\mathrm{p}}$ is the specific heat obtained by a differential scanning calorimeter (TA DSC Q20, USA) in argon. The measurement error of the electrical conductivity, the Seebeck coefficient and thermal conductivity were $\pm 5 \%, \pm 2 \%$ and $\pm 5 \%$, respectively. Ultrasonic Pulse-echo Method (Tektronix, TDS2022 USA) was used to measure the transverse velocity $\left(\nu_{\mathrm{t}}\right)$ and longitudinal velocity $\left(\nu_{1}\right)$ of the sample, and the elastic modulus was calculated by the following equations: $:^{36,37}$

$$
\begin{gathered}
G=d \nu_{\mathrm{t}}^{2} \\
E=d \frac{3 \nu_{\mathrm{t}}{ }^{2} \nu_{1}{ }^{2}-4 \nu_{\mathrm{t}}{ }^{2}}{\nu_{1}{ }^{2}-\nu_{\mathrm{t}}{ }^{2}} \\
\nu=\frac{E}{2 G}-1 \\
B=\frac{E}{3(1-2 \nu)}
\end{gathered}
$$

where $d$ is the density, $G$ is the shear modulus, $E$ is the Young's modulus, $\nu$ is the Poisson's ratio and $B$ is the bulk modulus. Vickers hardness was measured by microhardness instrument (Qness, Q10A+, Austria) with the main load of $2.0 \mathrm{~kg}$ and the dwell time of $10 \mathrm{~s}$.

\section{Results and discussion}

\subsection{Structure and morphology of $\beta$-NSA doped PPy}

FTIR spectra of pure PPy and $\beta$-NSA doped PPy is shown in Fig. 1. In the spectrum of pure PPy, the peaks at $882 \mathrm{~cm}^{-1}$ and 


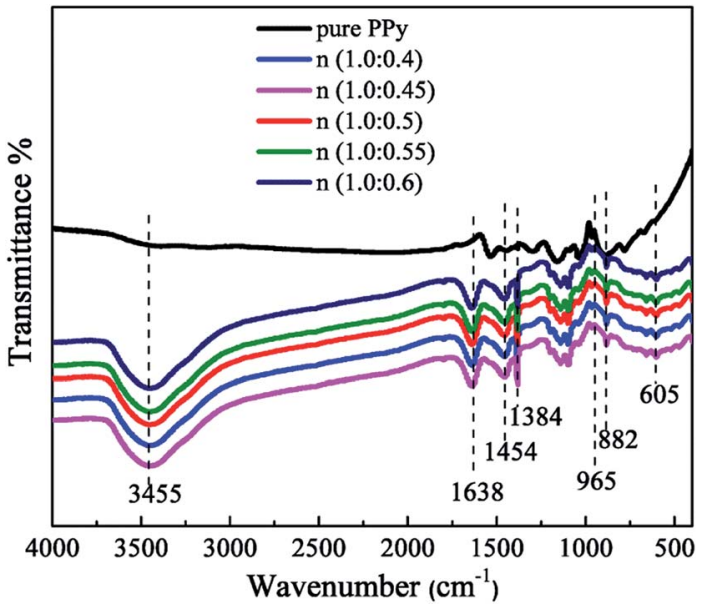

Fig. 1 FTIR spectra of pure PPy and PPy doped with various $\beta-N S A$ contents.

$965 \mathrm{~cm}^{-1}$ are attributed to the vibration of the $\mathrm{C}-\mathrm{H}$. The peaks located at $1384 \mathrm{~cm}^{-1}$ and $1454 \mathrm{~cm}^{-1}$ represent the $\mathrm{C}-\mathrm{N}$ and $\mathrm{C}=\mathrm{C}$ stretching vibration, respectively, which are also the characteristic peaks of pyrrole ring. The peak at $3455 \mathrm{~cm}^{-1}$ corresponds to the $\mathrm{N}-\mathrm{H}$ stretching vibration. ${ }^{38}$ Compared with the pure PPy, two peaks located at $605 \mathrm{~cm}^{-1}$ and $1638 \mathrm{~cm}^{-1}$ can be noticed in the spectra of the PPy/ $\beta$-NSA samples, which can be assigned to the characteristic vibrational peaks of $\mathrm{SO}_{3}{ }^{-}$and benzene ring, respectively. ${ }^{39}$ It can be concluded that the $\beta$-NSA has been doped into PPy successfully.

XRD analysis of the samples was showed in Fig. 2. For the pure PPy sample, there is only a broad characteristic peak at $24.5^{\circ}$ correspond to the $d$ spacing values of $3.6 \AA$, which originates from $\pi-\pi$ stacking of pyrrole rings, indicating that pure PPy is amorphous. ${ }^{40,41}$ However, three characteristic peaks of $\beta$ NSA doped PPy samples can be observed at about $7.4^{\circ}, 10.1^{\circ}$ and $24.5^{\circ}$, corresponding to the $d$ spacing values of $11.48,8.67$ and $3.4 \AA$, respectively. These $d$ values can be associated with the interlayer distance of pyrrole rings, pyrrole ring to $\mathrm{NSA}^{-}$ion dopant and $\pi-\pi$ stacking distance, ${ }^{42,43}$ which also can be ascribed to the crystallization peak of $\mathrm{PPy}^{44}$ And the peak

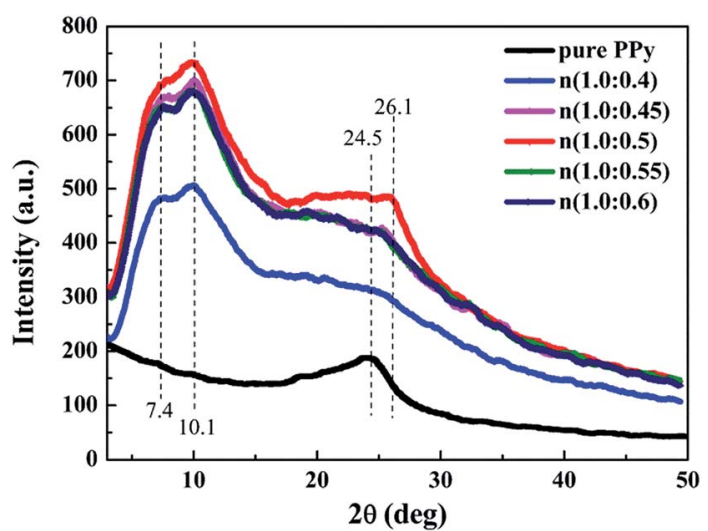

Fig. 2 XRD patterns of pure PPy and PPy doped with various $\beta$-NSA contents. associated to $\pi-\pi$ interaction shifted from $24.5^{\circ}$ to $26.1^{\circ}$ after doping $\beta$-NSA, which can be explained by the introduction of the large conjugated structure of $\beta$-NSA strengthened the $\pi-\pi$ interaction. ${ }^{43}$ The crystallinity $\left(X_{\mathrm{c}}\right)$ of the samples can be calculated according to the peak area of the crystalline $\left(A_{\mathrm{c}}\right)$ and noncrystalline $\left(A_{\mathrm{a}}\right)$ parts, $X_{\mathrm{c}}=A_{\mathrm{c}} /\left(A_{\mathrm{a}}+A_{\mathrm{c}}\right) .^{45}$ The crystalline peak of pure PPy is too weak to calculate $X_{\mathrm{c}}$, while the crystallinity of the $\beta$-NSA doped PPy samples are about $41 \%, 47 \%, 48 \%, 44 \%$ and $45 \%$, respectively. From the above analysis, it is implied that the crystalline of PPy improved greatly after doping $\beta$-NSA.

In order to further elucidate the influence on PPy molecular chain after doping $\beta$-NSA, the Raman spectrum of all samples was carried out and the results are depicted in Fig. 3. The Raman spectrum of pure PPy displays two peaks located at 1358 $\mathrm{cm}^{-1}$ (D peak) and $1570 \mathrm{~cm}^{-1}$ (G peak), which are related to the pyrrole ring and $\mathrm{C}=\mathrm{C}$ molecular chain stretching vibration of PPy, respectively ${ }^{46,47}$ And the calculated peak intensity ratio of D peak and G peak $\left(I_{\mathrm{D}} / I_{\mathrm{G}}\right)$ is 0.92 . As can be seen from the Raman spectrum, the peaks position of $\beta$-NSA doped PPy have no obvious shift compared with that of pure PPy. However, with the increasing of $\beta$-NSA, the peak intensity ratio $\left(I_{\mathrm{D}} / I_{\mathrm{G}}\right)$ appears significant change. When molar ratios of py to $\beta$-NSA increase from $1.0: 0.4$ to $1.0: 0.6$, the $I_{\mathrm{D}} / I_{\mathrm{G}}$ changes from 0.92 to 0.89 , $0.63,0.63,0.89$ and 0.88 . Compared with pure PPy, the peak intensity ratios of $\mathrm{D}$ peak and $\mathrm{G}$ peak $\left(I_{\mathrm{D}} / I_{\mathrm{G}}\right)$ are significantly reduced. The result indicates that the conjugated structure of PPy is enhanced, the order degree of molecular chain is increased, and the degree of defects is greatly decreased after doping $\beta$-NSA. ${ }^{29}$ With the augment of $\beta$-NSA, the growth of $I_{\mathrm{D}} / I_{\mathrm{G}}$ is mainly attributed to the steric hindrance effect of $\mathrm{NSA}^{-}$ion weakened conjugated structure of PPy. ${ }^{48}$

Fig. 4(a)-(e) presents the FESEM images of the pure PPy and PPy doped with various molar ratios of $\beta$-NSA. Fig. 4(a) shows the morphology of pure PPy, which exhibits irregular granular clusters. When the molar ratio of py to $\beta$-NSA is $1.0: 0.4$, not only the granular structure exists, but also lamellar structure (Fig. 4(b)) can be observed; when the molar ratio of py to $\beta$-NSA increases to $1.0: 0.45$, it shows lamellar structures instead of

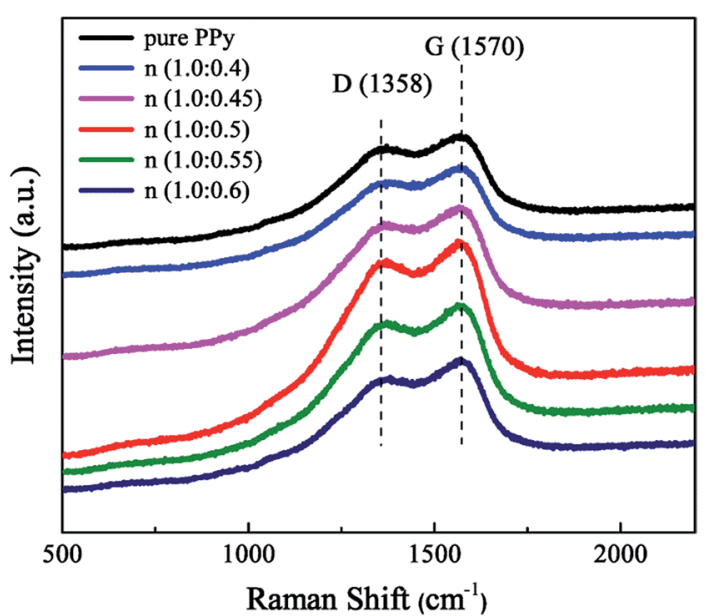

Fig. 3 Raman spectra of pure PPy and PPy doped with various $\beta$-NSA contents. 

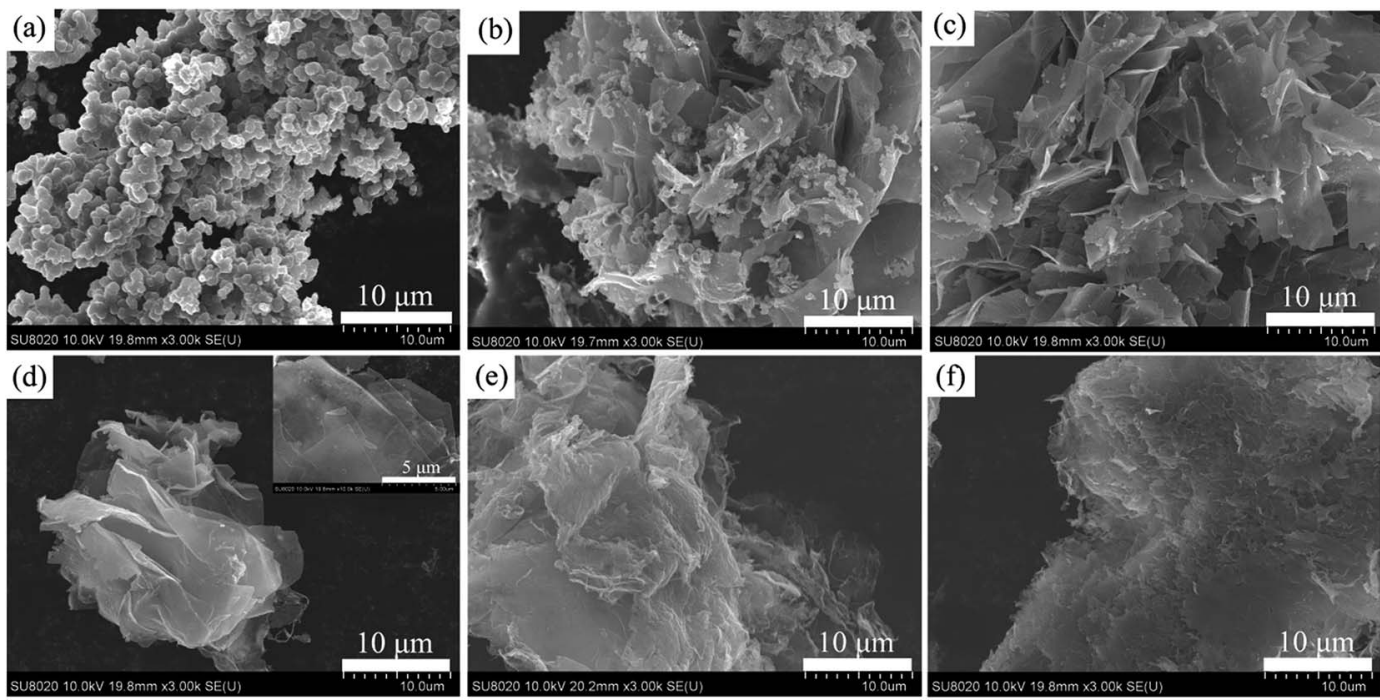

Fig. 4 FESEM images of all samples: (a) pure PPy and (b-f) PPy doped $\beta$-NSA with various molar ratios of py to $\beta$-NSA, (b) $1.0: 0.4$, (c) $1.0: 0.45$, (d) $1.0: 0.5$, (e) $1.0: 0.55$, (f) $1.0: 0.6$.

the former granular structure (Fig. 4(c)). When the molar ratio reaches $1.0: 0.5$, the lamellar area is enlarged and 8-9 layers stack together (Fig. 4(d)). And the sheet is very thin and nearly transparent, which can be seen from the illustration inset Fig. 4(d). As the molar ratio further increases, the number of stacked layers is extended to several tens of layers (Fig. 4(e) and (f)). Thus, it can be inferred that different concentration of $\beta$ NSA in the solution has a great impact on the morphology of PPy. Well known, $\beta$-NSA contains a polar functional group of sulfonic acid and a naphthyl with rich electronic properties due to its $\pi-\pi$ bond. It can greatly modify the conjugate of PPy molecular chain structure on account of the big conjugate structure of $\beta$-NSA. ${ }^{39,49}$ It is easy to form NSA micelles in aqueous solution due to the hydrophilic nature of NSA. Thus, it is reasonable to propose that NSA micelles might act as dopant and templates at the same time in the formation of PPy. ${ }^{35,50,51}$ When the amount of $\beta$-NSA is low, the formed NSA micelles are limited, only parts of the pyrrole monomers grew along the $\beta$ NSA micelles templates, thus granular and lamellar structures can coexist. When $\beta$-NSA reached a certain concentration, it can induce enough NSA micelles as templates for all the pyrrole monomers, thus only the lamellar structure can be observed. When the concentration of $\beta$-NSA is too high, the PPy sheets turned stacked together. However, the $\beta$-NSA doped PPy (or PANI) exhibited microtubular morphology when APS was used as oxidant, instead of $\mathrm{FeCl}_{3} .{ }^{52}$ It can be ascribed to the redox potential of APS is $2.05 \mathrm{~V}$, and the redox potential of $\mathrm{FeCl}_{3}$ is $0.77 \mathrm{~V}$, the oxidizing ability of the $\mathrm{FeCl}_{3}$ is weaker than APS, ${ }^{53}$ thus the polymerization rate of py monomer is slower, so that it preferred to form lamellar PPy rather than crinkle into tubular. Moreover, the concentration of py monomer is also important for the morphology. At a high concentration of py monomer, it turned to form granular morphology. ${ }^{54}$ And the concentration of py monomer in our work is low, this may also favored in producing lamellar PPy. In conclusion, the features of the dopant, the monomer concentration, and the type of oxidant, as well as polymerization conditions can affect the morphology of PPy. Both $\beta$-NSA and $\mathrm{FeCl}_{3}$ can effectively control the morphology of PPy to form a lamellar structure during the polymerization process. Based on the analysis of the FESEM images, a formation procedure of lamellar PPy is put forward in Fig. 5.

\subsection{Thermoelectric properties of $\beta$-NSA doped PPy}

Fig. 6(a)-(f) illustrates the electrical conductivity, Seebeck coefficient, thermal conductivity, power factor and $Z T$ of PPy doped with different molar ratios of $\beta$-NSA in the temperature range of 300-400 K. For all samples, as we can see from Fig. 6(a), the electrical conductivity is enhanced dramatically with the rising temperature, showing the characteristic of nondegenerate semiconductor transport behavior. At the same time, when the molar ratio of monomer py to $\beta$-NSA increased from $1.0: 0.4$ to $1.0: 0.6$, the electrical conductivity of PPy first increases and then decreases with the increment of $\beta$-NSA doping concentration and reaches the maximum when the molar ratio is $1.0: 0.5$. This phenomenon might be attributed to

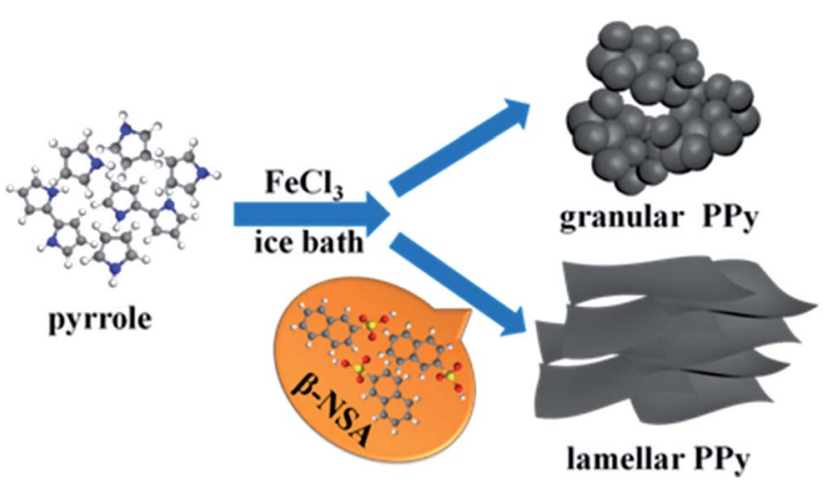

Fig. 5 Schematic illustration of the formation procedure of lamellar PPy. 

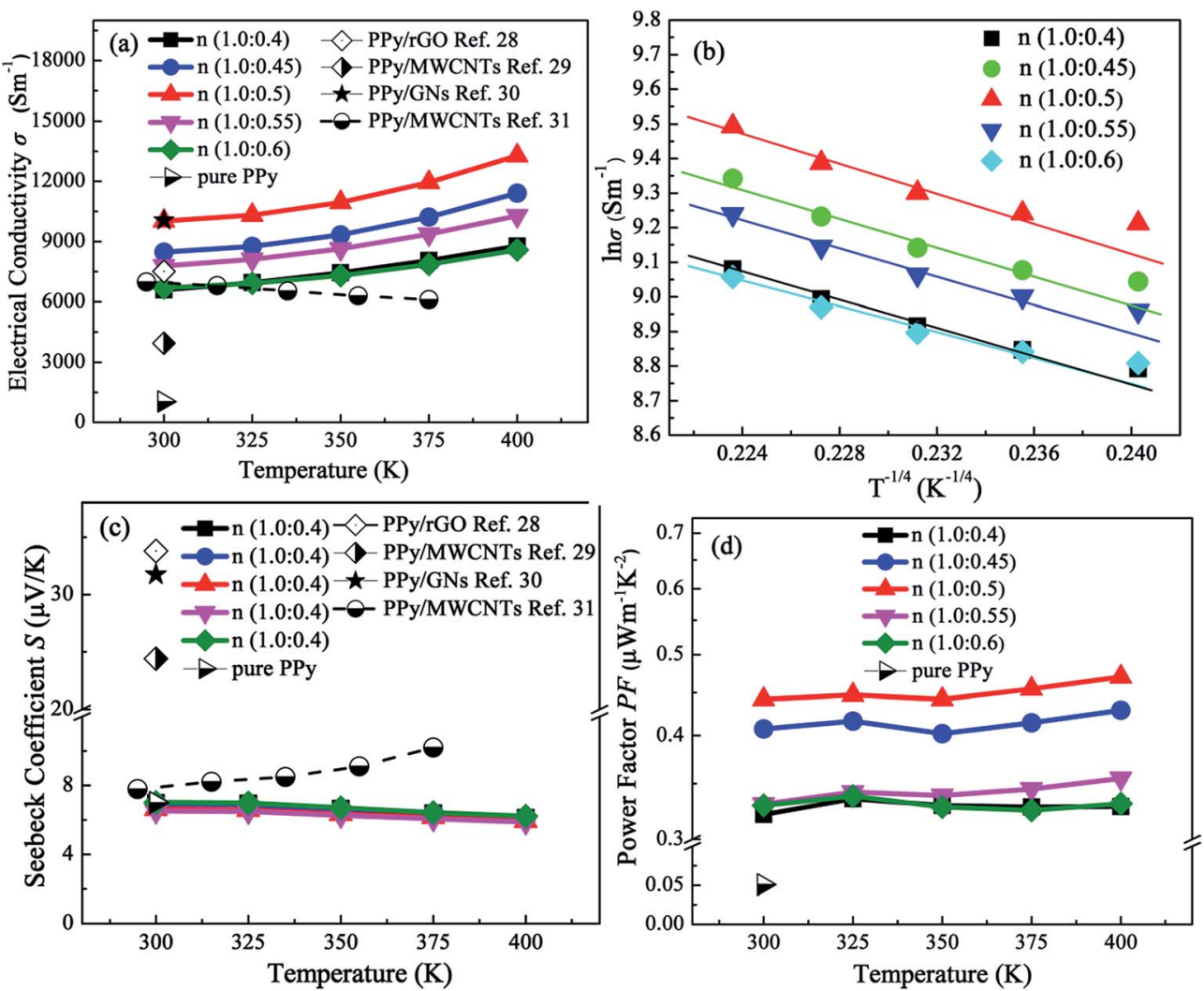

Fig. 6 Temperature dependence of thermoelectric properties of all samples, (a) electrical conductivity, (b) the plots of $\ln \sigma$ vs. $T^{-1 / 4}$, (c) Seebeck coefficient and (d) power factor.

the following two reasons: one is the large conjugated structure of $\beta$-NSA, and the other is the steric hindrance effect of $\mathrm{NSA}^{-}$ ion. $^{55}$ Firstly, with the increasing of $\beta$-NSA content, the enhancement of electrical conductivity is mainly originated from the large conjugated structure of $\beta$-NSA. According to the Raman spectra and XRD pattern, the decline of $I_{\mathrm{D}} / I_{\mathrm{G}}$ indicates that the degree of conjugation and the order of PPy molecular chain are strengthened after doping with $\beta$-NSA. In the meantime, the FESEM image shows that the microstructure of PPy transforms from granular to lamellar. The reinforcement of PPy molecular chain conjugation and the lamellar structure are favorable for the transport of carriers between interchain and intrachain, resulting in the increased electrical conductivity. When the molar ratio of py to $\beta$-NSA is $1.0: 0.5$, the $I_{\mathrm{D}} / I_{\mathrm{G}}$ dropped to a minimum value of 0.63 and the electrical conductivity achieves the maximum of $133 \mathrm{~S} \mathrm{~cm}^{-1}$ at $400 \mathrm{~K}$. The electrical conductivity of $\beta$-NSA doped PPy is about 10 times larger than that of pure $\mathrm{PPy}\left(10.34 \mathrm{~S} \mathrm{~cm}^{-1}\right)$ at room temperature. With the increasing of $\beta$-NSA, the anion steric hindrance effect of $\beta$-NSA plays a dominant role. Thus the degree of separation is aggravated and the degree of conjugation is weakened between the molecular chains of PPy which is consistent with the results of Raman analysis (namely $I_{\mathrm{D}} / I_{\mathrm{G}}$ increases). The carrier hopping will be hindered between molecular chains, which lead to the decrease of electrical conductivity. Compared with others reported PPy composite samples, the electrical conductivity of $\beta$ NSA doped PPy is also considerable high. ${ }^{28-31}$
The plots of $\ln \sigma$ versus $T^{-1 / 4}$ are shown in Fig. 6(b). It can be observed that $\ln \sigma$ is linearly related to $T^{-1 / 4}$, which is in accordance with Mott's 3D Variable Range Hopping (VRH) model, except that $\ln \sigma$ deviates linearly from $T^{-1 / 4}$ slightly at room temperature. The equation can be expressed as follows: $\sigma_{(T)}=\sigma_{0} \exp \left[-\left(T_{0} / T\right)^{1 / 4}\right]$, where $\sigma_{0}$ is a constant, $T_{0}$ is the Mott characteristic temperature, which can be calculated from the slope of the curve, $T$ is the Kelvin temperature. ${ }^{56}$

Fig. 6(c) displays the Seebeck coefficient of all samples. The Seebeck coefficient of all samples is positive, suggesting p-type conductive behavior. It is apparent that the Seebeck coefficient of all samples is similar to that of pure PPy $\left(6.995 \mu \mathrm{V} \mathrm{K}^{-1}\right)$, and the Seebeck coefficient does not change with the increasing of temperature and the doping amount of $\beta$-NSA. Because of the energy filtering effect at the interfaces between PPy and composite material, the Seebeck coefficient of PPy composite samples is high in comparison with doped with $\beta$-NSA. ${ }^{30,31}$

We know that the electrical conductivity is dependent on carrier mobility, which can be improved by the degree of crystallization of the polymer chain. A higher crystalline of the polymer resulted in a smaller barrier of carrier mobility within the intra-chain and the inter-chain. ${ }^{57,58}$ In general, the chains of conductive polymer are amorphous, showing the short-range order but long-range disorder. From the above analysis of FTIR, Raman spectra and FESEM image, when $\beta$-NSA was introduced into the polymerization system, $\beta$-NSA can not only act as soft template in the process of monomer polymerization 
to improve the microstructure of PPy, but also serve as dopant to heighten the conjugation degree of the molecular chain. As a result, both the carrier transition barrier and the degree of defects in the PPy chain are greatly reduced, which lead to a further improvement in the electrical conductivity.

Fig. 6(d) demonstrates the power factor $\left(S^{2} \sigma\right)$ of all the PPy samples. Because of much enhanced electrical conductivity by doping $\beta$-NSA, the power factor of PPy doped with $\beta$-NSA is strengthened to some extent and it reaches the maximum $0.47 \mu \mathrm{W} \mathrm{m} \mathrm{m}^{-1} \mathrm{~K}^{-2}$ when the molar ratio of py to $\beta$-NSA is $1.0: 0.5$, which is 9 times higher than that of pure PPy $\left(0.0506 \mu \mathrm{W} \mathrm{m}{ }^{-1} \mathrm{~K}^{-2}\right)$.

As shown in Fig. 7, the thermal conductivity of PPy increases with the rise of temperature, and increases with the increment of $\beta$-NSA doping amount. When the molar ratio of py to $\beta$-NSA is $1.0: 0.5$, the thermal conductivity obtained the maximum of $1.07 \mathrm{~W} \mathrm{~m}^{-1} \mathrm{~K}^{-1}$ at $400 \mathrm{~K}$, but the thermal conductivity of all the samples are comparable to pure PPy $\left(0.240 \mathrm{~W} \mathrm{~m}^{-1} \mathrm{~K}^{-1}\right)$ at room temperature. Generally speaking, the thermal conductivity of thermoelectric materials derives from phonons $\left(\kappa_{\mathrm{p}}\right)$ and electrons $\left(\kappa_{\mathrm{e}}\right) \cdot{ }^{59}$ However, the polymers with different crystalline, the contribution of the two parts to the thermal conductivity is different. According to Wiedemann-Franz's law: $\kappa_{\mathrm{e}}=L T \sigma$, where $L$ is the Lorentz factor, $\sigma$ is the electrical conductivity, and $T$ is the absolute temperature. ${ }^{60}$ For a typical conductive polymer, even the electrical conductivity reach up to $300 \mathrm{~S} \mathrm{~cm}^{-1}, \kappa_{\mathrm{e}}$ is almost negligible on account of quite low value. For instance, the study of PANI manifested that although the electrical conductivity increased more than nine orders of magnitude, the change of thermal conductivity was not significant. ${ }^{61}$ In addition, the thermal conductivity of organic thermoelectric materials is usually closely linked with the ordering degree of molecular chain arrangement and the charge carrier concentration, the higher degree of order, the greater thermal conductivity. The increase in thermal conductivity of PPy doped with $\beta$-NSA may be due to the following two reasons: on the one hand, the enhanced of order and conjugation of PPy chain makes phonons harder to scatter. On the other hand, after

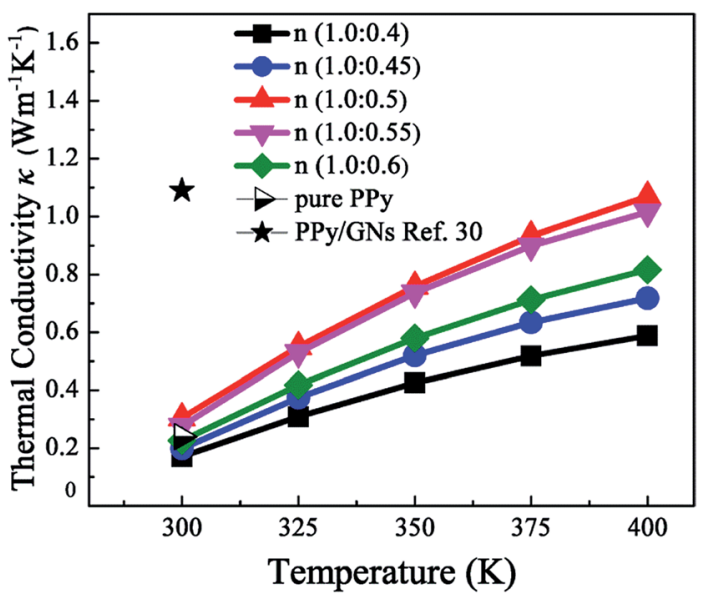

Fig. 7 Temperature dependence of the thermal conductivity of all samples and PPy/GNs ref. 30. doping $\beta$-NSA, the density of charge carrier increases so as to promote the heat transfer.

Fig. 8 depicts the $Z T$ values of PPy doped with different molar ratios of $\beta$-NSA as a function of temperature. The maximum $Z T$ value of $0.62 \times 10^{-3}$ is obtained when the molar ratio of py to $\beta$ NSA is $1.0: 0.45$ at $300 \mathrm{~K}$, which is approximately 10 times higher than that of pure PPy $\left(0.064 \times 10^{-3}\right)$. This is attributed to two aspects: firstly, the electrical conductivity is dramatically improved. Secondly, the thermal conductivity is maintained at a low level or even reduced at room temperature. Despite the electrical conductivity increases significantly at all test temperature range, the $Z T$ value at high temperature is not improved, because the thermal conductivity shows an upward trend and the Seebeck coefficient is not developed.

\subsection{Thermal stability of $\beta$-NSA doped PPy}

Thermal stability of conductive polymer is an important parameter, which determines the application temperature range. Therefore, TG analysis for all samples including pure $\beta$ NSA has been carried out from room temperature to $700{ }^{\circ} \mathrm{C}$ under heating rate of $10{ }^{\circ} \mathrm{C} \mathrm{min}^{-1}$ in nitrogen atmosphere and the result is displayed in Fig. 9. From the figure, it can be seen that $\beta$-NSA is particularly stable and does not lose weight before $550{ }^{\circ} \mathrm{C}$. And then it begins to decompose at $550{ }^{\circ} \mathrm{C}$ and the weight loss is about $40 \%$ between $550{ }^{\circ} \mathrm{C}$ and $700{ }^{\circ} \mathrm{C}$, indicating that the thermal stability of $\beta$-NSA is very good before $550{ }^{\circ} \mathrm{C}$. Besides, the TGA curves of pure PPy and PPy doped with $\beta$-NSA show a similar shape, suggesting that these samples display a similar degradation procedure. The initial weight loss up to $100{ }^{\circ} \mathrm{C}$ may stem from the removal of absorbed water. ${ }^{62}$ Compared with pure PPy, when the molar ratio is $1.0: 0.55$, the thermal stability of PPy is the best. The weight loss rate of PPy doped with $\beta$-NSA is slower under $410{ }^{\circ} \mathrm{C}$ and the weight loss is about $25 \%$, the weight loss rate is quicker above $410{ }^{\circ} \mathrm{C}$ and the weight loss is about $43 \%$ at $700{ }^{\circ} \mathrm{C}$. This is primarily attributed to the thermal decomposition property of $\beta$-NSA. The results declared that the thermal stability of PPy could strengthen in the temperature range of $\mathrm{RT}-400{ }^{\circ} \mathrm{C}$, and aggravate the degradation of PPy at high temperature $\left(>400{ }^{\circ} \mathrm{C}\right)$ after doping $\beta$-NSA.

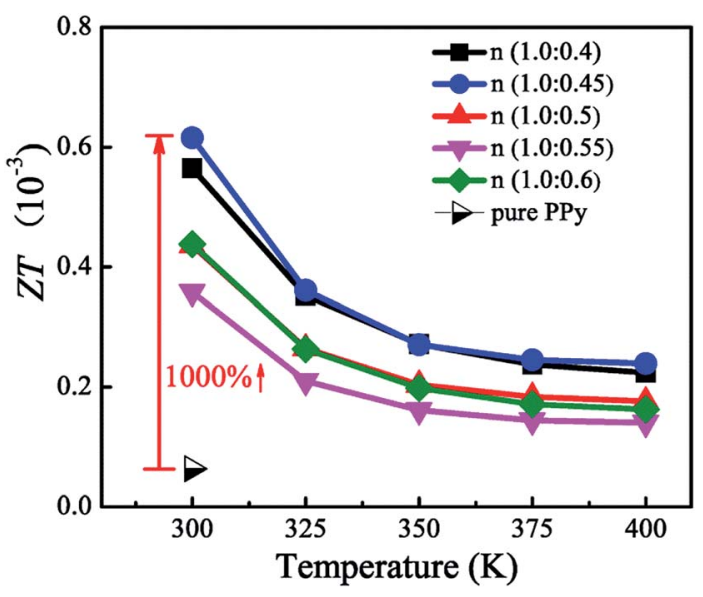

Fig. $8 Z T$ values of all samples as a function of temperature. 


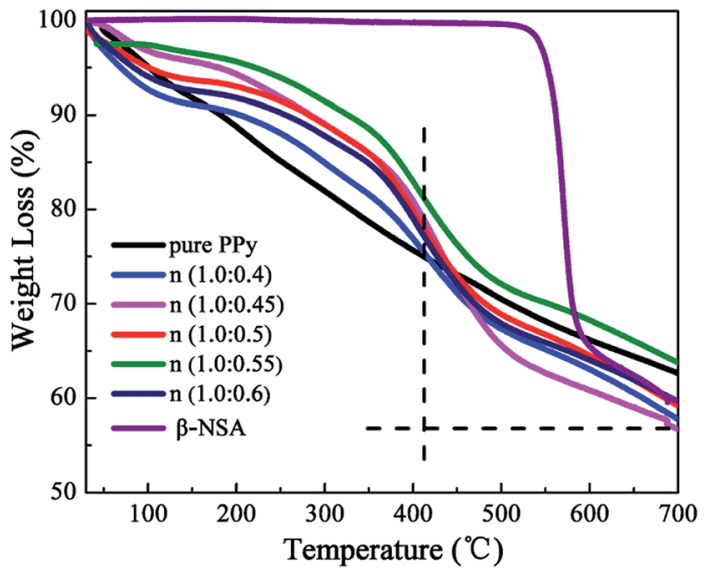

Fig. 9 TGA curves of pure PPy, $\beta$-NSA and PPy doped with various $\beta$ NSA contents.

\subsection{Mechanical properties of $\beta$-NSA doped PPy}

In addition to exploring the thermoelectric properties of PPy, we also studied the mechanical properties of PPy. The elastic modulus (bulk modulus, Yong's modulus and shear modulus) and Vickers hardness of the samples were carried out. It can be seen from the Fig. 10(a) that the elastic modulus of PPy is enhanced after doping $\beta$-NSA. And the Yong's modulus and shear modulus among the $\beta$-NSA doped PPy samples showed little difference, while the bulk modulus of the $\beta$-NSA doped PPy increased with the increasing of $\beta$-NSA. The enhanced elastic modulus is due to the microstructure of PPy changing from granular to lamellar and the enhancement of molecular chain arrangement tightness after doping $\beta$-NSA. And the molecular structure of $\beta$-NSA contains two benzene rings, which can further increase the rigidity of PPy when $\beta$-NSA are introduced into PPy chains. ${ }^{63}$

Besides, Vickers hardness is the parameters evaluating the ability of the material to resist plastic deformation, it was showed in Fig. 10(b). It can be found that the hardness of PPy increased firstly after doping $\beta$-NSA, and then decreased with higher doping level of $\beta$-NSA. The hardness of pure PPy is 15.4 MPa, while the maximum hardness of 31.1 MPa was obtained when the molar ratio of monomer py to $\beta$-NSA is $1.0: 0.45$, this is consistence with the thermoelectric properties of the samples. Compared with the pure PPy, the hardness of the $\beta$-NSA doped PPy was greatly improved, and it exhibited an enhanced ability to resist plastic deformation. This is due to the $\beta$-NSA template in favor of the formation of lamellar PPy, which possessed more regular and close arrangement of the molecular chains of PPy. The free volume of PPy can be promoted when excess $\mathrm{NSA}^{-}$ions were introduced into the main chain of PPy, resulting in a looser PPy backbone. ${ }^{64}$ Meanwhile, the wear resistance of the material is proportional to hardness. The higher the Vickers hardness, the better the wear resistance of the material. ${ }^{65}$

\section{Conclusions}

$\beta$-NSA doped lamellar polypyrrole (PPy) was successfully prepared via chemical oxidation polymerization method with varying the mole ratio of monomer py to $\beta$-NSA under ice bath $\left(<5{ }^{\circ} \mathrm{C}\right)$. $\beta$-NSA can not only improve the microstructure of PPy effectively due to the formation of $\beta$-NSA micelles in the solution which acted as template in the polymerization process, but also enhance the ordering of the molecular chains and the conjugated degree of the PPy owing to the large conjugated structure of $\beta$-NSA, which are both led to the increase of the electrical conductivity. Meanwhile, the thermal conductivity is also kept at a low level even decreased with the increase of doping amount at room temperature. So that the highest thermoelectric figure of merit $Z T$ is achieved $0.62 \times 10^{-3}$ with the molar ratio of monomer py to $\beta$-NSA of $1.0: 0.45$ at $300 \mathrm{~K}$, which is 10 times higher than that of pure PPy. Moreover, the thermal stability and mechanical properties of PPy are also enhanced greatly after doping $\beta$-NSA. These results implied that organic thermoelectric materials become promising candidates for the applications of low-cost and low-density thermoelectric materials. There is still much space for further improvement of the thermoelectric performance in comparison with other organic thermoelectric materials, especially the enhancement of the Seebeck coefficient.
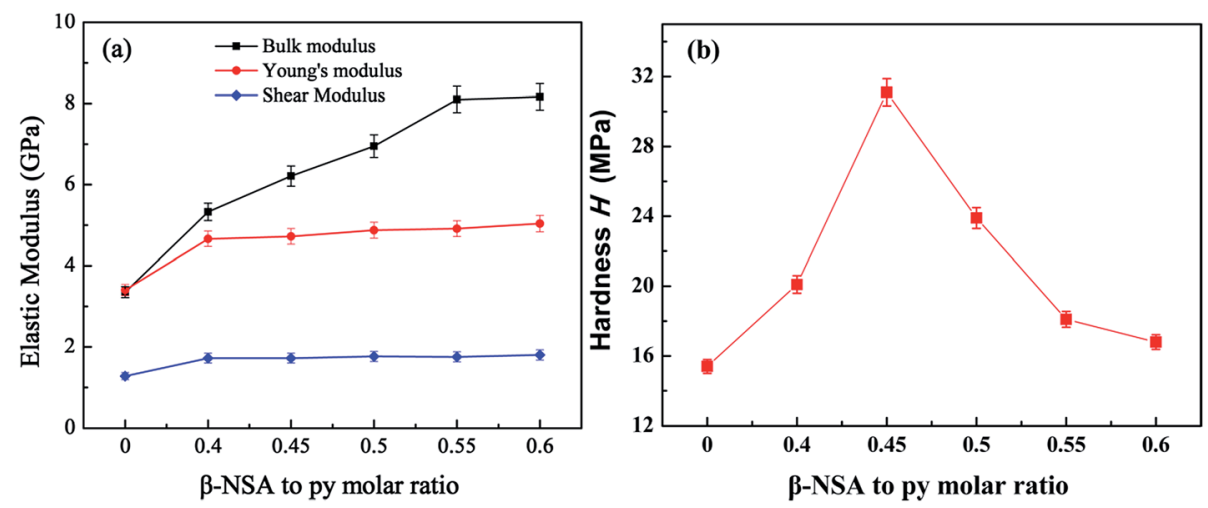

Fig. 10 Mechanical properties of all PPy samples, (a) elastic modulus, (b) hardness. 


\section{Acknowledgements}

The authors wish to acknowledge support from the National Basic Research Program of China (973 program) under project 2013CB632502, the Natural Science Foundation of China (Grant No. 51521001, 51632006) and the 111 Project of China (Grant No. B07040).

\section{Notes and references}

1 O. Bubnova and X. Crispin, Energy Environ. Sci., 2012, 5, 9345-9362.

2 Q. Zhang, Y. Sun, W. Xu and D. Zhu, Adv. Mater., 2014, 26, 6829-6851.

3 M. S. Dresselhaus, G. Chen, M. Tang, R. Yang, H. Lee, D. Z. Wang, Z. F. Ren, J. P. Fleurial and P. Gogna, Adv. Mater., 2007, 19, 1043-1053.

4 F. J. DiSalvo, Science, 1999, 285, 703-706.

5 L. E. Bell, Science, 2008, 321, 1457-1461.

6 T. M. Tritt, H. Boettner and L. Chen, MRS Bull., 2008, 33, 366-368.

7 D. K. C. MacDonald, Thermoelectricity: an introduction to the principles, Courier Corporation, 2006.

8 R. B. Aich, N. Blouin, A. Bouchard and M. Leclerc, Chem. Mater., 2009, 21, 751-757.

9 N. Toshima, Macromol. Symp., 2002, 186, 81-86.

10 J.-H. Bahk, H. Fang, K. Yazawa and A. Shakouri, J. Mater. Chem. C, 2015, 3, 10362-10374.

11 J. Zhao, D. Tan and G. Chen, J. Mater. Chem. C, 2017, 5, 4753.

12 N. Massonnet, A. Carella, O. Jaudouin, P. Rannou, G. Laval, C. Celle and J.-P. Simonato, J. Mater. Chem. C, 2014, 2, 12781283.

13 M. He, F. Qiu and Z. Lin, Energy Environ. Sci., 2013, 6, 13521361.

14 O. Bubnova, Z. U. Khan, A. Malti, S. Braun, M. Fahlman, M. Berggren and X. Crispin, Nat. Mater., 2011, 10, 429-433.

15 L. Wang, Q. Yao, H. Bi, F. Huang, Q. Wang and L. Chen, J. Mater. Chem. A, 2015, 3, 7086-7092.

16 Q. Yao, L. Chen, W. Zhang, S. Liufu and X. Chen, ACS Nano, 2010, 4, 2445-2451.

17 N. T. Kemp, A. B. Kaiser, C. J. Liu, B. Chapman, O. Mercier, A. M. Carr, H. J. Trodahl, R. G. Buckley, A. C. Partridge, J. Y. Lee, C. Y. Kim, A. Bartl, L. Dunsch, W. T. Smith and J. S. Shapiro, J. Polym. Sci., Part B: Polym. Phys., 1999, 37, 953-960.

18 J. Wu, Y. Sun, W. Pei, L. Huang, W. Xu and Q. Zhang, Synth. Met., 2014, 196, 173-177.

19 C. Bounioux, P. Diazchao, M. Campoyquiles, M. S. Martingonzalez, A. R. Goni, R. Yerushalmirozen and C. Muller, Energy Environ. Sci., 2013, 6, 918-925.

20 G. H. Kim, L. Shao, K. Zhang and K. P. Pipe, Nat. Mater., 2013, 12, 719-723.

$21 \mathrm{~J}$. Li, X. Tang, H. Li, Y. Yan and Q. Zhang, Synth. Met., 2010, 160, 1153-1158.

22 L. Li, F. Yan and G. Xue, J. Appl. Polym. Sci., 2004, 91, 303307.
23 Y. Fu and A. Manthiram, Chem. Mater., 2012, 24, 3081-3087. 24 S. Ye and J. Feng, ACS Appl. Mater. Interfaces, 2014, 6, 96719679.

25 A. T. Mane, S. D. Sartale and V. B. Patil, J. Mater. Sci.: Mater. Electron., 2015, 26, 8497-8506.

26 H. Xiao and S. Fu, CrystEngComm, 2014, 16, 2097-2112.

27 M. Culebras, B. Uriol, C. M. Gomez and A. Cantarero, Phys. Chem. Chem. Phys., 2015, 17, 15140-15145.

28 Z. Zhang, G. Chen, H. Wang and W. Zhai, J. Mater. Chem. C, 2015, 3, 1649-1654.

29 H. Song, K. Cai, J. Wang and S. Shen, Synth. Met., 2016, 211, 58-65.

30 L. Wang, F. Liu, C. Jin, T. Zhang and Q. Yin, RSC Adv., 2014, 4, 46187-46193.

31 J. Wang, K. Cai, S. Shen and J. Yin, Synth. Met., 2014, 195, 132-136.

32 Y. Shen and M. Wan, J. Polym. Sci., Part A: Polym. Chem., 1997, 35, 3689-3695.

33 Y. Shen and M. Wan, Synth. Met., 1998, 96, 127-132.

34 R. O. Akinyeye, M. Sekota, P. Baker and E. I. Iwuoha, Fullerenes, Nanotubes, Carbon Nanostruct., 2006, 14, 49-55.

35 J. Liu and M. Wan, J. Mater. Chem., 2001, 11, 404-407.

36 H. Ledbetter, N. V. Frederick and M. W. Austin, J. Appl. Phys., 1980, 51, 305-309.

37 J. E. Bailey, Nature, 1979, 280, 176.

38 T. Wu and S. Lin, J. Polym. Sci., Part A: Polym. Chem., 2006, 44, 6449-6457.

39 J. Y. Lee, D. Y. Kim and C. Y. Kim, Synth. Met., 1995, 74, 103106.

40 L. F. Warren, J. A. Walker, D. P. Anderson, C. G. Rhodes and L. J. Buckley, J. Electrochem. Soc., 1989, 136, 2286-2295.

41 K. J. Wynne and G. B. Street, Macromolecules, 1985, 18, 23612368.

42 K. Cheah, M. Forsyth and V. T. Truong, Synth. Met., 1998, 94, 215-219.

43 P. Carrasco, H. Grande, M. Cortazar, J. M. Alberdi, J. Areizaga and J. A. Pomposo, Synth. Met., 2006, 156, 420-425.

44 P. P. Jeeju, S. J. Varma, P. A. F. Xavier, A. M. Sajimol and S. Jayalekshmi, Mater. Chem. Phys., 2012, 134, 803-808.

45 R. R. Hegde, M. G. Kamath and A. Dahiya, Polymer Crystallinity, 2004, available online, http://web.utk.edu/ $\sim$ mse/Textiles/Polymer\%20Crystallinity.htm.

46 B. Saner, S. A. Gursel and Y. Yurum, Fullerenes, Nanotubes, Carbon Nanostruct., 2013, 21, 233-247.

47 Z. Gu, C. Li, G. Wang, L. Zhang, X. Li, W. Wang and S. Jin, J. Polym. Sci., Part B: Polym. Phys., 2010, 48, 1329-1335.

48 S. Gupta, J. Raman Spectrosc., 2008, 39, 1343-1355.

49 Z. Zhang, L. Zhu, Y. Ma, Y. Huang and G. Li, Analyst, 2013, 138, 1156-1166.

50 Y. Yang, J. Liu and M. Wan, Nanotechnology, 2002, 13, 771773.

51 L. Xia, Z. Wei and M. Wan, J. Colloid Interface Sci., 2010, 341, 1-11.

52 M. Wan, J. Huang and Y. Shen, Synth. Met., 1999, 101, 708711.

53 H. Ding, M. Wan and Y. Wei, Adv. Mater., 2007, 19, 465-469. 
54 P. Jha, S. P. Koiry, V. Saxena, P. Veerender, A. K. Chauhan, D. K. Aswal and S. K. Gupta, Macromolecules, 2011, 44, 4583-4585.

55 L. Fang, L. Zhao, X. Liang, H. Xiao and L. Qian, J. Appl. Polym. Sci., 2016, 133, 43759.

56 B. F. M. A. Davis, Electric process in nom-crystalline materials, Clarendon Press, 2012.

57 Y. Zhao, G. S. Tang, Z. Z. Yu and J. S. Qi, Carbon, 2012, 50, 3064-3073.

58 Q. Wang, Q. Yao, J. Chang and L. D. Chen, J. Mater. Chem., 2012, 22, 17612-17618.

59 G. D. Mahan and M. Bartkowiak, Appl. Phys. Lett., 1999, 74, 953-954.
60 M. Jonson and G. D. Mahan, Phys. Rev. B: Condens. Matter Mater. Phys., 1980, 21, 4223-4229.

61 H. Yan, N. Sada and N. Toshima, J. Therm. Anal. Calorim., 2002, 69, 881-887.

62 H. Arami, M. Mazloumi, R. Khalifehzadeh, S. H. Emami and S. K. Sadrnezhaad, Mater. Lett., 2007, 61, 4412-4415.

63 R. J. Roberts, R. C. Rowe and P. York, Powder Technol., 1991, 65, 139-146.

64 O. Uzun, N. Basman, C. Alkan, U. Kolemen and F. Yilmaz, Polym. Bull., 2010, 66, 649-660.

65 A. Faltermeier, C. Reicheneder, P. Romer, A. Castrolaza and P. Proff, Journal of Orofacial Orthopedics / Fortschritte der Kieferorthopädie, 2014, 75, 334-344. 